\title{
NON-GAUSSIAN HALO BIAS AND FUTURE GALAXY SURVEYS
}

\author{
Carmelita Carbone, ${ }^{1}$ Licia Verde, ${ }^{1,2,3}$ and Sabino Matarrese ${ }^{4}$ \\ Received 2008 June 22; accepted 2008 July 21; published 2008 August 13
}

\begin{abstract}
We forecast constraints on primordial non-Gaussianity achievable from forthcoming surveys by exploiting the scale-dependent halo bias introduced on large scales by non-Gaussian initial conditions. We explore the performance of exploiting both the shape of the galaxy power spectrum on large scales and the cross-correlation of galaxies with cosmic microwave background maps through the integrated Sachs-Wolfe effect. We find that future surveys can detect primordial non-Gaussianity of the local form with a non-Gaussianity parameter $\left|f_{\mathrm{NL}}\right|$ of order unity. This is particularly exciting because, while the simplest single-field slow-roll models of inflation predict a primordial $\left|f_{\mathrm{NL}}\right| \ll 1$, this signal sources extra contributions to the effective $f_{\mathrm{NL}}$ of large-scale structures that are expected to be above our predicted detection threshold.
\end{abstract}

Subject headings: cosmology: theory — galaxies: clusters: general — galaxies: halos —

large-scale structure of universe

\section{INTRODUCTION}

Tests of deviations from Gaussian initial conditions offer an important window into the very early universe and a powerful test for the mechanism which generated primordial perturbations. While standard single-field slow-roll models of inflation lead to small departures from Gaussianity, nonstandard scenarios allow for a larger non-Gaussianity (NG) level (see, e.g., Bartolo et al. 2004 and references therein). The standard observables to constrain NG are the cosmic microwave background (CMB) and the large-scale structure (LSS) of the universe.

Traditionally, the most popular method to detect primordial NG has been to measure the bispectrum or the three-point function of the CMB (Verde et al. 2000; Komatsu et al. 2005; Yadav \& Wandelt 2008), as the LSS bispectrum is sensitive to primordial NG only at high redshift (Verde et al. 2000; Scoccimarro et al. 2004; Sefusatti \& Komatsu 2007; Cooray 2006; Pillepich et al. 2007). A powerful technique is based on the abundance (Matarrese et al. 2000; Verde et al. 2001; LoVerde et al. 2008; Robinson \& Baker 2000; Robinson et al. 2000) and clustering (Grinstein \& Wise 1986; Matarrese et al. 1986) of rare events such as density peaks, as they trace the tail of the underlying distribution. These predictions have been tested against $N$-body simulations (Kang et al. 2007; Grossi et al. 2007; Dalal et al. 2007). Dalal et al. (2007) and Matarrese \& Verde (2008) showed that primordial NG affects the clustering of dark matter halos inducing a scale-dependent large-scale bias. Here we argue that this effect could be used to constrain NG through the integrated Sachs-Wolfe (ISW) effect (Sachs \& Wolfe 1967) and through the shape of the galaxy power spectrum on large scales. We forecast how future galaxy surveys could constrain Gaussianity via this "halo bias" effect. We find that constraints from surveys which provide a large sample of galaxies or galaxy clusters over a volume comparable to the Hubble volume (e.g., DES, PanSTARRS, LSST, EUCLID,

\footnotetext{
${ }^{1}$ Institute of Space Sciences (CSIC-IEEC), UAB, Barcelona 08193, Spain; carbone@ieec.uab.es.

${ }^{2}$ ICREA; verde@ieec.uab.es.

${ }^{3}$ Department of Astrophysical Sciences, Princeton University, Ivy Lane, Princeton, NJ

${ }^{4}$ Dipartimento di Fisica "G. Galilei," Università degli Studi di Padova and INFN, Sezione di Padova, via Marzolo 8, 35131 Padova, Italy; sabino.matarrese@pd.infn.it.
}

ADEPT) are competitive with CMB bispectrum constraints achievable with an ideal CMB experiment. Bartolo et al. (2005) showed that even when the primordial $f_{\mathrm{NL}}$ is tiny, the evolution of perturbations on super-Hubble scales yields extra contributions to the effective $f_{\mathrm{NL}}$ relevant for the LSS, which are configuration- and redshift-dependent. These contributions are of amplitude comparable to the forecasted errors and can therefore no longer be neglected. Along the way, we offer physical insights in the findings of Matarrese \& Verde (2008, hereafter MV08) and explain the connections to the approach of Dalal et al. (2007); we show that only the formalism of MV08 can be used to correctly handle the nonlinear contributions to primordial NG.

\section{NON-GAUSSIAN HALO BIAS IN CONTEXT}

MV08 generalized to NG initial conditions results and techniques developed in the 1980s (Grinstein \& Wise 1986; Matarrese et al. 1986) to relate the clustering properties of the collapsed structures (halos) to those of the underlying matter distribution for Gaussian initial conditions. This approach yields an analytic expression for the bias of dark matter halos for NG models in which the primordial bispectrum of the potential is the dominant higher order correlation and has a general form. The starting point of MV08 is to consider the expression for the correlation function of regions above a high threshold in the general NG case, which has the form (Grinstein \& Wise 1986; Matarrese et al. 1986)

$$
\xi_{h, M}(r)=-1+\exp [X(r)]
$$

where $X$ is a complicated expression that depends on all the $n$-point correlations of the underlying density field filtered on the mass scale $M$ and on the threshold height (see MV08 for details). One interprets the region above high thresholds as halos, and for large separations $r$ (small values of $X$ ), expands the exponential to first order and considers only terms up to the three-point correlation function. Finally, one Fourier-transforms to get an approximated expression for the halo power spectrum in NG models. For NG of the type (Salopek \& Bond 
1990; Gangui et al. 1994; Verde et al. 2000; Komatsu \& Spergel 2001)

$$
\Phi=\phi+f_{\mathrm{NL}}\left(\phi^{2}-\left\langle\phi^{2}\right\rangle\right)
$$

where $\Phi$ denotes Bardeen's gauge-invariant potential ${ }^{5}$ and $\phi$ denotes a Gaussian random field, the halo power spectrum has the form

$$
P_{h}(k, z)=\frac{\delta_{c}^{2}(z) P_{\delta \delta}(k, z)}{\sigma_{M}^{4} D^{2}(z)}\left[1+4 f_{\mathrm{NL}} \delta_{c}(z) \alpha(k)\right] .
$$

Here $\alpha(k)$ is the quantity in Figure 3 of MV08, which is $\propto 1 / k^{2}$ at large scales; $\sigma_{M}^{2}$ is the mass variance linearly extrapolated to $z=0 ; \delta_{c}(z)=\Delta_{c}(z) / D(z)$, with $\Delta_{c}(z)$ the linear overdensity for spherical collapse (weakly dependent on $z$ in nonEinstein-de Sitter cosmologies) and $D(z)$ the linear growth factor of density fluctuations, normalized to $D(0)=1$.

The halo Lagrangian bias $b_{\mathrm{L}}$ (all correlations and peaks considered here are those of the initial density field) is then $b_{\mathrm{L}, h}(z, M) \simeq b_{\mathrm{L}, h}^{\mathrm{G}}(z, M)\left[1+2 f_{\mathrm{NL}} \delta_{c}(z) \alpha(k)\right]$, with $b_{\mathrm{L}, h}^{\mathrm{G}}(z, M) \simeq$ $\delta_{c}(z) /\left[\sigma_{M}^{2} D(z)\right]$. Making the standard assumption that halos move coherently with dark matter, one gets the Eulerian bias as $b_{\mathrm{E}}=1+b_{\mathrm{L}}$, so that

$$
b_{h}^{f_{\mathrm{NL}}}=1+\frac{\delta_{c}\left(z_{f}\right)}{\sigma_{M}^{2} D\left(z_{o}\right)}\left[1+2 f_{\mathrm{NL}} \delta_{c}\left(z_{f}\right) \alpha(k)\right],
$$

where, following Catelan et al. (1998) we have made explicit the dependence on both halo formation redshift $z_{f}$ and observation redshift $z_{o}$. This expression for the NG halo bias is scaledependent and increases rapidly at large scales. The identification of halos with density peaks is valid for rare (massive) halos. The above expression for the Gaussian Lagrangian halo bias is approximate, a more accurate expression being (Efstathiou et al. 1988; Cole \& Kaiser 1989; Mo \& White 1996)

$$
b_{\mathrm{L}, h}^{\mathrm{G}}\left(z_{o}, M, z_{f}\right)=\frac{1}{D\left(z_{o}\right)}\left[\frac{\delta_{c}\left(z_{f}\right)}{\sigma_{M}^{2}}-\frac{1}{\delta_{c}\left(z_{f}\right)}\right] .
$$

For objects that did not undergo recent mergers, $z_{f} \gg z_{o}$, the bias is well approximated by equation (4). Equation (4) also applies to the case $z_{f} \approx z_{o}$ (rapid mergers) for $\delta_{c}^{2} \gg \sigma_{M}^{2}$, i.e., large $M$ and/or high $z_{f}$.

Equation (4) can be rewritten as $b_{\mathrm{L}, h}^{f_{\mathrm{NL}}}=b_{\mathrm{L}, h}^{\mathrm{G}}+\Delta b$, where

$$
\Delta b=2 f_{\mathrm{NL}} \delta_{c}\left(z_{f}\right)\left[b_{h}^{\mathrm{G}}\left(z_{o}, M, z_{f}\right)-1\right] \alpha(k) .
$$

One may note that for $f_{\mathrm{NL}}$ large and negative, equation (3) would yield $b_{h}^{f_{\mathrm{NL}}}$ and $P_{h}(k)$ negative on large enough scales. This is a manifestation of the breakdown of the approximations made; i.e., all correlations of higher order than the bispectrum were neglected (for large NG this truncation may not hold) and the exponential in equation (1) was linearly expanded. This however could be easily corrected for, remembering that the $P(k)$ obtained in equation (3) is the Fourier transform of $X$, the argument of the exponential. One would then compute the halo correlation function using equation (1) and Fourier-transforming back to obtain the halo power spectrum.

Dalal et al. (2007) and Slosar et al. (2008) use the peakbackground split to obtain an equation similar to equation (4). In particular, while Dalal et al. (2007) relies on the spherical

\footnotetext{
${ }^{5}$ Following the LSS convention, here $\Phi$ is linearly extrapolated at $z=0$.
}

collapse model (and thus on the standard Press-Schechter [Press \& Schechter 1974] approach), Slosar et al. (2008) extend and reformulate it so that it relies on the extended Press-Schechter (EPS) approach and on the universality of the mass function, and can be obtained for any mass function, even one that is a fit to $N$-body simulations. The advantage of their formulation over that of Dalal et al. (2007) is that they can include the description of the effect of halo mergers. Although the effect of halo merger can be analytically described only for the PressSchechter mass function, they argue that the scaling of the correction for mergers could be calibrated from $N$-body simulations. Let us note that their derivation can only be carried out for NG of local type. The formulation of MV08 is instead more general: the EPS approach, halo mergers, and mass functions, which are better fit to $\mathrm{N}$-body simulations than the standard Press-Schechter one, can be readily taken into account by substituting $b_{\mathrm{L}, h}^{\mathrm{G}}$ in equation (5) by the peak-background split bias $b_{\mathrm{L}, h}^{\mathrm{G}}=-n^{-1} \partial n / \partial \delta_{c}$, where $n$ is the halo mass function for the Gaussian case, which could be given, e.g., by the Sheth \& Tormen (1999) formula or by a fit to simulations. In particular, equation (5) is replaced by

$$
\begin{aligned}
b_{\mathrm{L}, h}^{\mathrm{G}}\left(z_{o}, M, z_{f}\right)= & \frac{1}{D\left(z_{o}\right)}\left[\frac{q \delta_{c}\left(z_{f}\right)}{\sigma_{M}^{2}}-\frac{1}{\delta_{c}\left(z_{f}\right)}\right] \\
& +\frac{2 p}{\delta_{c}\left(z_{f}\right) D\left(z_{o}\right)}\left[1+\left(\frac{q \delta_{c}^{2}\left(z_{f}\right)}{\sigma_{M}^{2}}\right)^{p}\right]^{-1},
\end{aligned}
$$

where $q$ and $p$ account for nonspherical collapse and are fit to $N$-body simulations yielding $q \sim 0.75, p=0.3$ (Sheth \& Tormen 1999; Sheth et al. 2001). The correction for nonspherical collapse also applies to the NG correction to the halo bias:

$$
\Delta b=2 q^{\prime} f_{\mathrm{NL}} \delta_{c}\left(b_{h}^{\mathrm{G}}-1\right) \alpha(k),
$$

where $q^{\prime}$ can be calibrated to $N$-body simulations and is found to be $q^{\prime} \approx 0.8$ (M. Grossi et al., in preparation). We stress here that it is very important to be able to account for general nonlocal and scale-dependent NG characterized by a given bispectrum of $\Phi$. Indeed, as shown by Bartolo et al. (2005) there are extra contributions that come in at the same level as the primordial signal: in other words the primordial contribution is enhanced for LSS, yielding configuration- and redshift-dependent contributions to the effective $f_{\mathrm{NL}}$ which cannot be neglected. The NG bias formula of MV08 is fully general and can easily account for any nonconstant $f_{\mathrm{NL}}$.

\section{METHOD}

Let us start with two preliminary considerations.

Halos versus galaxies.-The theory developed in MV08 and above describes the clustering properties of halos, but we observe galaxies. Different galaxy populations occupy dark matter halos following different rules. If we think in the halo-model (Cooray \& Sheth 2002; Scoccimarro et al. 2001; Peacock \& Smith 2000; Smith et al. 2003) framework, at very large scales (those relevant for this analysis), only the "two halo" contribution matters and the details of the halo occupation distribution of galaxies (the "one halo" term) is unimportant. In particular, the galaxy population known as "luminous red galaxies" (LRG) is known to be old and free from recent merger activity. For this population, the MV08 modeling should offer a good description. Emission-line galaxies on the other hand may be affected by recent mergers and their $b_{h}^{\mathrm{G}}$ may need to be modified (see $\S 2$ ). As we discuss below, with this modi- 
fication, the effect of uncertainties in the Gaussian bias enters in our estimates only through the shot-noise contribution to the signal-to-noise ratio.

Detection versus measurement.-One should make the important distinction between "detection" and "measurement." To compute the statistical significance of a detection we need to compute the significance of deviations from the null hypothesis: in particular, the fiducial model used in the calculation has $f_{\mathrm{NL}}=0$ and the error bars are also computed assuming the null hypothesis $f_{\mathrm{NL}}=0$. However, to carry out a measurement of $f_{\mathrm{NL}} \neq 0$, the model and the error bars must be computed as functions of $f_{\mathrm{NL}}$. Here we will report forecasts for detection of $f_{\mathrm{NL}}$.

We consider two probes: the large-scale galaxy power spectrum and the ISW effect. In both cases, since we will consider tracers of rare halos, we will set $z_{f}=z_{o}$, yielding a possibly conservative estimate of $f_{\mathrm{NL}}$ errors.

\subsection{Forecasts from the Shape of the Large-Scale Power Spectrum}

In the Fisher matrix approach to error forecasts we can write

$$
\ln \mathcal{L}=-\frac{1}{2} \frac{[\Delta P(k)]^{2}}{\sigma_{P}^{2}}=-\frac{1}{2} \frac{\left[P(k) 4 \delta_{c}(z) \alpha(k) f_{\mathrm{NL}}\right]^{2}}{\sigma_{P}^{2}}
$$

where we have assumed a Gaussian likelihood. ${ }^{6}$ An estimate of the error in $f_{\mathrm{NL}}, \sigma_{f_{\mathrm{NL}}}(k)$, at a given $k$ is given by

$$
\frac{1}{\sigma_{f_{N L}}(k)^{2}}=\frac{\partial^{2}|\ln \mathcal{L}|}{\partial f_{\mathrm{NL}}^{2}}=\frac{16 P^{2} \alpha(k)^{2} \delta_{c}^{2}}{\sigma_{P}^{2}} .
$$

The relative error in $P$ for a shell in $k$-space of width $\Delta k$ and for a survey with effective volume $V_{\text {eff }}$ is

$$
\left(\frac{\sigma_{P}}{P}\right)^{2}=\frac{2}{4 \pi k^{2} \Delta k V_{\mathrm{eff}} /\left(2 \pi^{3}\right)}
$$

with $V_{\text {eff }}=V[1+1 /(\bar{n} P)], V$ the survey volume, and $\bar{n}$ the mean density of galaxies. If shot noise is subdominant (i.e., $\bar{n} P \gg 1), V_{\text {eff }}=V$.

The total error for a $k$-range from $k_{\min }$ to $k_{\max }$ is

$$
\frac{1}{\sigma_{f_{N L}^{2}}^{2}}=\frac{8}{2 \pi^{2}} \frac{\Delta_{c}(z)^{2}}{D(z)^{2}} V_{\text {eff }} \int_{k_{\min }}^{k_{\max }} \alpha(k)^{2} k^{2} d k
$$

If we divide the survey in redshift slices centered around $z_{i}$ then the error obtained combining different redshift slices (if uncorrelated $)^{7}$ is

$$
\frac{1}{\sigma_{f_{N L}}^{2}}=\sum_{i} \frac{1}{\sigma_{f_{N L}}\left(z_{i}\right)^{2}}
$$

We set $k_{\max }$ to be $0.03 h \mathrm{Mpc}^{-1}$ and $k_{\text {min }}$ to be greater than the $2 \pi / V^{1 / 3}$ where $V$ is the volume of the shell considered $(\Delta z=0.1$ which is larger than standard photometric redshift errors). Conservatively, we do not consider that scales larger than $k_{\min }$ can be used by cross-correlating different shells. The effect of NG alters the broadband behavior of the $P(k)$ on very

\footnotetext{
${ }^{6}$ While strictly speaking the distribution of $P(k)$ is non-Gaussian, this is a standard assumption in Fisher-matrix-based approaches.

${ }^{7}$ The correlation between shells is small at $k \ll 0.03 h \mathrm{Mpc}^{-1}(P(k) \sim 1 / k)$ and $\Delta z=0.1\left(200 \mathrm{Mpc} h^{-1}\right.$ at $\left.z=0.8\right)$.
}

large scales, which is unaffected by the precision with which the radial positions of the galaxies are measured. Thus, we can treat photometric and spectroscopic surveys on the same footing. The requirement of surveying a large volume of the universe and sampling highly biased galaxies to beat shot noise, which is a key point for BAO surveys, is also a bonus for constraining primordial NG. In particular, for the $k_{\min }$ we use we find that $P_{g}\left(k_{\min }\right) \simeq P_{g}\left(k=0.2 h \mathrm{Mpc}^{-1}\right)$; thus the shotnoise requirement for $\mathrm{BAO}$ surveys of $\bar{n} P(k=0.2 h$ $\left.\mathrm{Mpc}^{-1}\right)>1$ implies that for all scales of interest here $\bar{n} P \gg$ 1 . We have checked that our results do not change if we impose $\bar{n} P \sim 3$. While for BAO surveys measuring redshift accurately is crucial (Seo \& Eisenstein 2003; Blake \& Bridle 2005), for this application is not important.

\subsection{Forecasts from the Integrated Sachs-Wolfe Effect}

The ISW effect probes the largest cosmological scales. As the NG effect goes $\propto 1 / k^{2}$ on large scales, this is a promising probe of NG. Here we follow Afshordi (2004) to quantify the significance of a detection of $f_{\mathrm{NL}}$ through the estimate of the cross-correlation between the ISW effect and the LRG galaxy distribution. For a galaxy survey with the mean comoving density distribution $n_{c}(r)$ as a function of the comoving distance $r$, in the Limber approximation the expected cross-correlation in spherical harmonic space can be written as

$$
C_{g T}(\ell)=\frac{2 T}{\int d r r^{2} n_{c}(r)} \int d r n_{c}(r) P_{\Phi, g}(k)
$$

where $k=(\ell+1 / 2) / r$ and $\Phi^{\prime}$ is the derivative of the gravitational potential with respect to the conformal time. The expected dispersion in the cross-correlation signal is $\Delta C_{g T}^{2}(\ell) \simeq$ $C_{g g}(\ell) C_{T T}(\ell)\left[f_{\text {sky }}(2 \ell+1)\right]^{-1}$, where $f_{\text {sky }}$ is the fraction of sky covered by the survey and we assumed a small cross-correlation signal, i.e., $C_{g T}^{2}(\ell) \ll C_{g g}(\ell) C_{T T}(\ell)$.

For a galaxy distribution biased according to equation (4), dividing the survey in redshift shells and following the same procedure of $\S 3$, the error in each shell at redshift $z$ for a given $\ell$ is

$$
\sigma_{f_{\mathrm{NL}}}^{-2}=\frac{\gamma\left[H(z) D(z)(d / d z)[(1+z) D(z)] P_{\delta \delta}(k, 0) \Delta b(k, z)\right]^{2} r^{2} \delta r}{(2 l+1)^{3} C_{T T}(\ell)\left[P_{\mathrm{G}}(k, z)+n_{c}(r)^{-1}\right]},
$$

where $\quad \gamma=8 f_{\text {sky }}\left(3 T H_{0}^{2} \Omega_{m 0} / c^{3}\right)^{2}, \quad k \equiv(l+1 / 2) / r, \quad \delta r=$ $[c / H(z)] \Delta z, \Delta b$ is equation (6) in the limit $f_{\mathrm{NL}}=1$, and $P_{\mathrm{G}}$ denotes the galaxy power spectrum in the Gaussian case. We impose $k_{\min }$ to be greater than the largest mode that can be

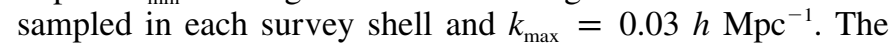
total error is obtained summing up the latter expression on all $\ell \leq 200$ and integrating over the minimum and maximum redshift of each survey.

For future large-scale galaxy surveys, in the case of nondominant shot noise, we obtain $\Delta f_{\mathrm{NL}}=5.7,10.8$, and 9.8 for LSST, EUCLID, and ADEPT, respectively.

\section{RESULTS AND DISCUSSION}

Here we present forecasts on $f_{\mathrm{NL}}$ constraints for forthcoming and future surveys. The surveys and their specifications are reported in Table 1 , with the $1 \sigma$ error on $f_{\mathrm{NL}}$ from the shape of the galaxy power spectrum. The errors on $f_{\mathrm{NL}}$ have been normalized by the correction factor for nonspherical collapse 
TABLE 1

Galaxy Surveys Considered

\begin{tabular}{|c|c|c|c|c|}
\hline Survey & $z$ Range & $\begin{array}{l}\text { Square } \\
\text { Degrees }\end{array}$ & $\begin{array}{l}\text { Mean Galaxy } \\
\text { Density } \\
\left(h \mathrm{Mpc}^{-1}\right)^{3}\end{array}$ & $\Delta f_{\mathrm{NL}} / q^{\prime} \mathrm{LSS}$ \\
\hline $\begin{array}{l}\text { SDSS LRGs } \\
\text { SDS }\end{array}$ & $0.16<z<0.47$ & $7.6 \times 10^{3}$ & $1.36 \times 10^{-4}$ & 37 \\
\hline BOSS & $0<z<0.7$ & $10^{4}$ & $2.66 \times 10^{-4}$ & 18.8 \\
\hline WFMOS low $z$ & $0.5<z<1.3$ & $2 \times 10^{3}$ & $4.88 \times 10^{-4}$ & 15 \\
\hline WFMOS high $z$ & $2.3<z<3.3$ & $3 \times 10^{2}$ & $4.55 \times 10^{-4}$ & 17.5 \\
\hline ADEPT & $1<z<2$ & $2.8 \times 10^{4}$ & $9.37 \times 10^{-4}$ & 1.7 \\
\hline EUCLID & $0<z<2$ & $2 \times 10^{4}$ & $1.56 \times 10^{-3}$ & 1.9 \\
\hline DES & $0.2<z<1.3$ & $5 \times 10^{3}$ & $1.85 \times 10^{-3}$ & 8.4 \\
\hline PanSTARRS & $0<z<1.2$ & $3 \times 10^{4}$ & $1.72 \times 10^{-3}$ & 3.7 \\
\hline LSST & $0.3<z<3.6$ & $3 \times 10^{4}$ & $2.77 \times 10^{-3}$ & 0.7 \\
\hline
\end{tabular}

$q^{\prime} \approx 0.8$. The number of galaxies and the Gaussian bias enter this signal-to-noise calculation only through the contribution to the error due to shot noise. The reported numbers are not dominated by shot noise. This signal-to-noise calculation indicates that the halo clustering approach to primordial NG is in principle more promising than the ISW one: the ISW signal is weighted at $z \lesssim 1$, when dark energy dominates, while the effect of NG grows with redshift. However, the two approaches are affected by different systematics and should be considered complementary.

It is worth comparing the constraints on primordial NG achievable from the large-scale halo clustering with those achievable with the small-scale galaxy bispectrum. A comparison with Sefusatti \& Komatsu (2007) shows that halo-clustering constraints are a factor of 3 stronger than bispectrum ones. The bispectrum, however, through its dependence on the $k$-space configuration, can be used to discriminate among different forms of NG. The CMB bispectrum for an ideal experiment can yield constraints of $\Delta f_{\mathrm{NL}}=$ a few (Yadav et al. 2007).
Table 1 indicates that constraints on $f_{\mathrm{NL}} \sim 1$ are achievable with future surveys, making it a highly competitive technique. Let us stress that it is important to be able to account for general nonlocal NG features characterized by a given bispectrum of $\Phi$. In fact, extra contributions to the bispectrum with a specific shape and redshift dependence unavoidably come into play at the level of $f_{\mathrm{NL}} \sim$ a few (Bartolo et al. 2005), i.e., well above the detection threshold for forthcoming and proposed surveys, thus opening up the possibility to measure them.

While this work was being completed we became aware of Afshordi \& Tolley (2008) and McDonald (2008). Our results are in good agreement with theirs.

C. C. is supported through a Beatrix de Pinos grant. L. V. is supported by FP7-PEOPLE-2007-4-3-IRG 202182 and CSIC I3 grant 200750I034. L. V. thanks W. Hu, M. Grossi, and E. Branchini for fruitful discussions. S. M. acknowledges partial support by ASI contract I/016/07/0 “COFIS" and ASI contract Planck LFI Activity of Phase E2.

\section{REFERENCES}

Afshordi, N. 2004, Phys. Rev. D, 70, 083536

Afshordi, N., \& Tolley, A. J. 2008, Phys. Rev. D, submitted (arXiv:0806.1046)

Bartolo, N., Komatsu, E., Matarrese, S., \& Riotto, A. 2004, Phys. Rep., 402, 103

Bartolo, N., Matarrese, S., \& Riotto, A. 2005, J. Cosmol. Astropart. Phys., 10, 010

Blake, C., \& Bridle, S. 2005, MNRAS, 363, 1329

Catelan, P., Lucchin, F., Matarrese, S., \& Porciani, C. 1998, MNRAS, 297, 692

Cole, S., \& Kaiser, N. 1989, MNRAS, 237, 1127

Cooray, A. 2006, Phys. Rev. Lett., 97, 261301

Cooray, A., \& Sheth, R. 2002, Phys. Rep., 372, 1

Dalal, N., Dore, O., Huterer, D., \& Shirokov, A. 2007, preprint (arXiv: 0710.4560)

Efstathiou, G., Frenk, C. S., White, S. D. M., \& Davis, M. 1988, MNRAS, 235,715

Gangui, A., Lucchin, F., Matarrese, S., \& Mollerach, S. 1994, ApJ, 430, 447

Grinstein, B., \& Wise, M. B. 1986, ApJ, 310, 19

Grossi, M., Dolag, K., Branchini, E., Matarrese, S., \& Moscardini, L. 2007, MNRAS, 382, 1261

Kang, X., Norberg, P., \& Silk, J. 2007, MNRAS, 376, 343

Komatsu, E., \& Spergel, D. N. 2001, Phys. Rev. D, 63, 063002

Komatsu, E., Spergel, D. N., \& Wandelt, B. D. 2005, ApJ, 634, 14

LoVerde, M., Miller, A., Shandera, S., \& Verde, L. 2008, J. Cosmol. Astropart. Phys., 04, 014

Matarrese, S., Lucchin, F., \& Bonometto, S. A. 1986, ApJ, 310, L21

Matarrese, S., \& Verde, L. 2008, ApJ, 677, L77 (MV08)
Matarrese, S., Verde, L., \& Jimenez, R. 2000, ApJ, 541, 10

McDonald, P. 2008, preprint (arXiv:0806.1061)

Mo, H. J., \& White, S. D. M. 1996, MNRAS, 282, 347

Peacock, J. A., \& Smith, R. E. 2000, MNRAS, 318, 1144

Pillepich, A., Porciani, C., \& Matarrese, S. 2007, ApJ, 662, 1

Press, W. H., \& Schechter, P. 1974, ApJ, 187, 425

Robinson, J., \& Baker, J. E. 2000, MNRAS, 311, 781

Robinson, J., Gawiser, E., \& Silk, J. 2000, ApJ, 532, 1

Sachs, R. K., \& Wolfe, A. M. 1967, ApJ, 147, 73

Salopek, D. S., \& Bond, J. R. 1990, Phys. Rev. D, 42, 3936

Scoccimarro, R., Sefusatti, E., \& Zaldarriaga, M. 2004, Phys. Rev. D, 69, 103513

Scoccimarro, R., Sheth, R. K., Hui, L., \& Jain, B. 2001, ApJ, 546, 20

Sefusatti, E., \& Komatsu, E. 2007, Phys. Rev. D, 76, 083004

Seo, H.-J., \& Eisenstein, D. J. 2003, ApJ, 598, 720

Sheth, R. K., Mo, H. J., \& Tormen, G. 2001, MNRAS, 323, 1

Sheth, R. K., \& Tormen, G. 1999, MNRAS, 308, 119

Slosar, A., Hirata, C., Seljak, U., Ho, S., \& Padmanabhan, N. 2008, preprint (arXiv:0805.3580)

Smith, R. E., et al. 2003, MNRAS, 341, 1311

Verde, L., Jimenez, R., Kamionkowski, M., \& Matarrese, S. 2001, MNRAS, 325,412

Verde, L., Wang, L., Heavens, A. F., \& Kamionkowski, M. 2000, MNRAS, 313,141

Yadav, A. P. S., Komatsu, E., \& Wandelt, B. D. 2007, ApJ, 664, 680

Yadav, A. P. S., \& Wandelt, B. D. 2008, Phys. Rev. Lett., 100, 181301 